\title{
Incoherent neutron-scattering determination of hydrogen content: Theory and modeling
}

\author{
R. C. Perego a) and M. Blaauw ${ }^{\text {b) }}$ \\ Interfaculty Reactor Institute, University of Technology Delft, Mekelweg 15, 2629 JB Delft, The Netherlands
}

(Received 12 November 2004; accepted 2 May 2005; published online 23 June 2005)

\begin{abstract}
Hydrogen concentrations of 0 up to $350 \mathrm{mg} / \mathrm{kg}$ in a titanium alloy have been determined at National Institute of Standards and Technology (NIST) with neutron incoherent scattering (NIS) and with cold neutron prompt gamma activation analysis. The latter is a well-established technique, while the former was demonstrated earlier at NIST by counting the neutrons scattered under $45^{\circ}$ or $60^{\circ}$ and establishing a linear relationship between hydrogen concentration and count rate. In this paper, it is shown that the NIS response is complicated by the dependence of the apparent hydrogen scattering cross section on the sample temperature and the hydrogen chemical binding. It is demonstrated that detection of scattered neutrons at two different angles as well as a more complex theoretical approach (i.e., including Monte Carlo modeling with the free-gas model) are required to correctly interpret NIS measurements. As a result, the process of matching standards and samples becomes less critical. @ 2005 American Institute of Physics. [DOI: 10.1063/1.1940141]
\end{abstract}

\section{INTRODUCTION}

Hydrogen, the most abundant element, is often present as impurity in metals and alloys and can affect their mechanical properties. For example, titanium and its alloys, as used in jet engine compressor blades, embrittles due to the presence of hydrogen even at very low concentrations, in the order of $100-150 \mathrm{mg} / \mathrm{kg} .{ }^{1}$ Therefore it is of utmost importance to be able to determine very small hydrogen concentrations in this type of materials. At the National Institute of Standards and Technology (NIST) neutron incoherent scattering (NIS) has been developed as an independent technique, in addition to prompt gamma neutron activation analysis (PGNAA), for the determination of the hydrogen concentration in a titanium alloy ${ }^{2,3}$ in the process of reference material certification. In NIS, a white beam of cold neutrons impinges on the sample and the scattered neutrons are then detected under a few angles. The main element that is targeted in NIS is hydrogen, due to its large incoherent scattering cross section, which is 46 times larger than the coherent scattering cross section ( 80.3 vs $1.76 \mathrm{~b}$ ). ${ }^{4}$ The scattering cross section of hydrogen for slow neutrons is usually at least one order of magnitude higher than the scattering cross section of other elements, ${ }^{4}$ which results in a high sensitivity for hydrogen. In the early work, the neutron scattering was regarded as isotropic and was measured in one forward direction only (in some cases at $45^{\circ}$ and in others at $60^{\circ}$ ). Polypropylene foils were employed as standards for hydrogen. The results obtained agreed both with PGNAA measurements and with the concentrations expected from the synthesis procedure, indicating that the technique was suitable for the determination of the hydrogen concentration in the titanium alloy studied. The technique, which is denoted

\footnotetext{
a)Electronic mail: raffaella.perego@radiochemie.de

${ }^{b)}$ Fax: +31-15-2788303; electronic mail: m.blaauw@tnw.tudelft.nl
}

"isotropic NIS" in this paper, has more recently been applied elsewhere for the measurement of hydrogen concentration in zirconium alloys. 5

However, NIS measurements at more than one angle on the same titanium samples measured at NIST (Ref. 6) revealed that the isotropic approach to analyze NIS data is too simplistic: the observed hydrogen scattering cross section depends on the rigidity of the hydrogen chemical binding as well as on the related thermal motion of the hydrogen atoms in the samples. If the hydrogen is not rigidly bound, the scattering is anisotropic in the laboratory system, the anisotropy varying with hydrogen binding and temperature. These features could be employed to extract more information from the NIS experiments by measuring the scattering under various angles; on the other hand they could produce an unexpected bias in the information obtained in simplistic NIS determinations. ${ }^{6}$

The scope of the work described in this paper is to further develop the NIS technique by employing a more sophisticated theoretical model, which takes into account the hydrogen binding and the thermal motions of the scattering atoms by relating both to the "effective mass" of the hydrogen. Since the method proposed employs the free-gas model as theoretical base, the technique will be denoted "free-gas NIS," as opposed to isotropic NIS.

\section{EXPERIMENT}

\section{A. Sample preparation}

Titanium alloy specimens of $10 \times 10 \times 1 \mathrm{~mm}^{3}$ with nominal hydrogen concentrations of 0-, 92.3-, 208-, 238-, 306-, 275-, and 375-mg/kg hydrogen were prepared at NIST by a direct reaction method employing a Ti-6Al-4V alloy as starting material. ${ }^{7}$ The hydrogen mass fractions are expressed as relative to the titanium alloy mass. 


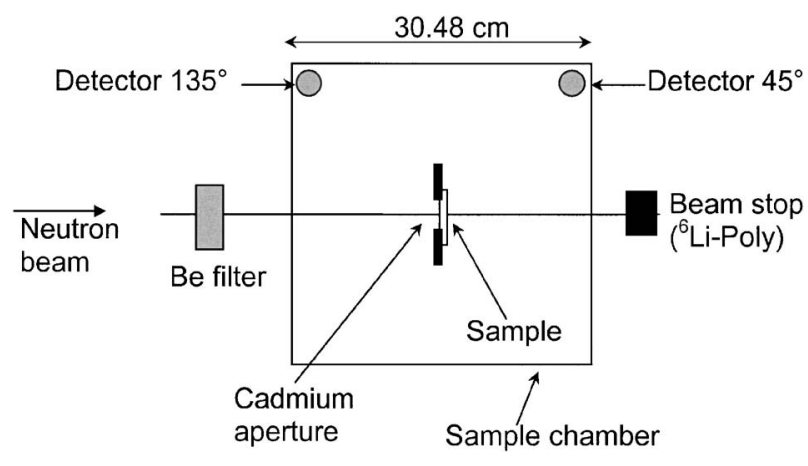

FIG. 1. The free-gas NIS experimental arrangement.

\section{B. Cold neutron prompt gamma activation analysis (CNPGAA)}

The hydrogen concentration of all titanium samples, except the $275 \mathrm{mg} / \mathrm{kg}$, was determined by CNPGAA in 1998 as described in Ref. 6. In 2002 all concentrations were redetermined.

\section{NIS}

The NIS measurements were performed at the neutron optics facility on the NG0 beam line at the Cold Neutron Research Facility of the NIST Centre for Neutron Research. A white beam of cold neutrons with an average wavelength of $0.4 \mathrm{~nm}$ was used. In order to decrease the thermal neutron fraction of the incident beam, a 50.8-mm-thick beryllium crystal at room temperature filtered the incident beam before the sample. The neutron fluence rate at the sample position was approximately $6.6 \times 10^{11} \mathrm{~m}^{-2} \mathrm{~s}^{-1}$.

The room-temperature sample was placed in a 30.48$\mathrm{cm}^{3}$ aluminum chamber with a sample holder, consisting of 0.5 -mm-thick cadmium foil elements screwed onto an aluminum frame, with a circular sample aperture of 0.9$\mathrm{mm}$-diameter. The collimated beam hit the sample after passing through a 5 -mm-diameter aperture in a $50.8-\mathrm{mm}^{2}$ Teflon window. After hitting the sample, it exited through a window of the same dimensions at the far side and was stopped by a ${ }^{6} \mathrm{Li}$-polymer foil placed behind the chamber. The scattered neutrons were detected with two cylindrical $9.525-\mathrm{cm}-$ long 40-bars ${ }^{3} \mathrm{He}$ proportional counters oriented perpendicular to the main plane of the arrangement and fixed at two corners of the chamber, i.e., at scattering angles of $45^{\circ}$ and $135^{\circ}$. The counters were collimated with cadmium plates $7.62 \mathrm{~cm}$ long, leaving an aperture of $2.54 \mathrm{~cm}$. The neutron flux was monitored continuously. In Fig. 1 the experimental geometry is depicted.

The standard measuring sequence for the titanium samples was background measurement, blank, hydrogen-intitanium samples, blank, and background again. Typical count rates were in the order of $100 \mathrm{~s}^{-1}$. Several series of such experiments were performed. The sample homogeneity was tested by changing the sample orientation, i.e., the samples were measured with both faces facing the neutron beam alternatingly.

The raw data for each sample, i.e., numbers of counts recorded at intervals ranging from $10 \mathrm{~s}$ to $1 \mathrm{~min}$, were cor- rected for the background and normalized to the signal of the blank titanium sample. Poisson statistics were propagated throughout.

\section{THEORY}

Hydrogen at low concentrations in Ti alloys may be considered randomly distributed, implying that the total scattering cross section gives rise to incoherent scattering. In the experiments described here, a cold neutron beam is employed, for which the neutron wavelength is larger than the crystal lattice dimensions of the metal alloy samples and coherent neutron scattering by the matrix may therefore be excluded as well. In addition, since the neutron energy is less than any of the $h \nu$ of molecular vibrations, inelastic scattering is not occurring either, since no energy can be transferred to the vibrations and the interaction is thus with the whole molecule as a rigid body. ${ }^{8}$ This implies that kinetic energy and momentum are conserved and the scattering is thus elastic (also known as quasielastic in the neutron-scattering community), and that classical mechanics apply. In short, the hydrogen atoms are considered as a free gas in the metal lattice. The direct influence of the chemical binding, as well as its indirect effect through the thermal motion of the atoms, is taken into account using an effective mass in conjunction with the free-gas model.

\section{A. The influence of chemical binding}

According to Fermi ${ }^{8}$ the scattering cross section of an atom of mass $M$ [amu] rigidly bound to an infinite mass, $\sigma_{\text {bound }}$, and of the free atom, $\sigma_{\text {free }}$, are related by the following expression:

$$
\frac{\sigma_{\text {bound }}}{\sigma_{\text {free }}}=\left(\frac{1+M}{M}\right)^{2} .
$$

Beckurts and Wirtz ${ }^{9}$ give the following expression for atoms that are neither free nor rigidly bound:

$$
\frac{M_{\mathrm{eff}}}{1+M_{\mathrm{eff}}} \frac{1+M}{M}=\sqrt{\frac{\sigma\left(M_{\mathrm{eff}}\right)}{\sigma_{\text {free }}}},
$$

where $M_{\text {eff }}$ is the effective mass of the scattering atoms with atomic mass $M$, and $\sigma\left(M_{\text {eff }}\right)$ is their scattering cross section. This expression results from a remark by Fermi that "the ratio of the bound-atom scattering cross section to the freeatom scattering cross section is just the square of the ratio of the corresponding reduced masses, ${ }^{, 10}$ In the case of hydrogen, where $M=1$,

$$
\frac{2 M_{\mathrm{eff}}}{1+M_{\mathrm{eff}}}=\sqrt{\frac{\sigma\left(M_{\mathrm{eff}}\right)}{\sigma_{0}}} .
$$

In the case of $M_{\text {eff }}=\infty$, Eq. (3) reduces to Eq. (1).

\section{B. The influence of thermal motion}

At room temperature the scattering atoms may not be at rest. If the velocities due to thermal motion are comparable to the velocities of the incoming neutron, the probability of the neutron being scattered increases significantly with the time the neutron spends in the scattering material. If the neu- 
tron velocity $v$ is low, a $1 / v$ relation is observed between the apparent scattering cross section and the neutron velocity. If the neutron velocity is much higher than the velocities of the scattering atoms, but not high enough to break chemical bonds, the apparent scattering cross section is constant as a function of $v$ and identical to the scattering cross section as commonly tabulated. A description of the phenomena is provided by the "free-gas model" applied in Monte Carlo codes such as Monte Carlo $N$-particle code ${ }^{11}$ (MCNP) where it is employed as the best possible approximation for any material for which the complete scattering kernel $S(Q, \omega)$ is unknown. In addition, the binding of hydrogen is taken into account by assuming effective scattering masses larger than unity. Increased binding implies increased scattering cross section and lowered velocities due to thermal motion. More detail on this approach is given further down.

\section{The anisotropy of the scattering}

The neutron scattering described is isotropic in the center-of-mass system. In the laboratory system, however, it is anisotropic, the anisotropy being a function of the effective mass of the scatterer and the temperature of the sample. In the ideal case of a scatterer initially at rest

$$
\langle\cos (2 \theta)\rangle=\frac{2}{3 M_{\mathrm{eff}}},
$$

where $2 \theta$ is the angle between the initial neutron direction and the final neutron direction. However, in the free-gas model, the scattering particle is not at rest: as the neutron velocity decreases or the scatterer's temperature increases, the scattering becomes more isotropic.

\section{Monte Carlo modeling: The BUDA program}

Up to the present time, no analytical expression has been found to describe the angular distribution of the scattered neutrons in the free-gas model. However, Monte Carlo software to model the scattering process was written specifically for the NIS measurements by employing the free-gas model. ${ }^{12}$ This solution was preferred to the use of the MCNP code, since the latter is less suitable for the modeling of purely theoretical scatterers.

The program simulates free-gas NIS experiments by computing self-shielding factors for objects defined beforehand. The self-shielding factor is defined as the number of absorbed neutrons in the object, divided by the product of object volume, macroscopic absorption cross section, and neutron flux density in the neutron field, which would be the expected capture rate in the beam in the case of infinite dilution. This definition is applied both to objects in the neutron beam (the scatterers) as to objects outside the neutron beam that interact only with scattered neutrons (the detectors).

Inputs for the program are the geometric data on the experiment, the neutron velocity distribution (in the case of the Maxwell-Boltzmann velocity distribution characterized by the temperature of the neutron beam), as well as the mac- roscopic scattering and absorption cross sections, the temperature, and the (effective) masses for the constituents of each object.

The macroscopic absorption and scattering cross sections $(\Sigma)$ for each element $x$ are obtained from the following equation:

$$
\Sigma_{x}=\frac{\rho_{x} N_{\mathrm{Av}}}{M_{x}} \sigma_{x}\left[\mathrm{~cm}^{-1}\right],
$$

where $N_{\mathrm{Av}}$ is Avogadro's number, $M_{x}$ is the atomic mass, $\sigma_{x}$ is the microscopic cross section in $\mathrm{cm}^{2}$, and $\rho_{x}\left[\mathrm{~g} / \mathrm{cm}^{3}\right]$ is the element density in the system analyzed:

$$
\rho_{x}=f_{x} \rho_{\mathrm{tot}}\left[\mathrm{g} \mathrm{cm}^{-1}\right],
$$

$f_{x}$ being the element fraction and $\rho_{\text {tot }}$ the density of the scatterer as a whole.

First, each neutron is drawn with a velocity $v$ according to the velocity distribution of the neutron source used. In the case of the experiments discussed in this paper, the source is the NG0 beam line at the Cold Neutron Research Facility of the National Institute for Standards and Technology (NIST) Centre for Neutron Research and the velocity distribution was determined experimentally. ${ }^{13}$

Then, the neutron just drawn is followed to the surface of the first object in its path. The scattering interaction frequency $I_{s}(v)$ of the scattering material is determined by summing the interaction frequencies of its atomic constituents $I_{s i}(v)$.

$$
I_{s}(v)=\sum_{i} I_{s i}(v)
$$

These individual interaction frequencies $I_{s i}(v)$ are obtained from the atomic scattering cross sections $\sigma_{s i}(v)$, their density $N_{i}$ in the material, and a correction factor for the free-gas effect, with the free-gas model formula

$$
I_{s i}(v)=\sigma_{s i} N_{i}\left\{\frac{v_{M}}{\sqrt{\pi}} e^{-\left(v / v_{M}\right)^{2}}+\left(\frac{1}{2} \frac{v_{M}^{2}}{v}+v\right) \operatorname{erf}\left(\frac{v}{v_{M}}\right)\right\},
$$

where $\sigma_{s i}$ is obtained with Eq. (2) and $v_{m}$ is the most likely velocity of the Maxwell-Boltzmann velocity distribution of the scatterer given by

$$
v_{M}=v_{0} \sqrt{\frac{T}{293.6}} \sqrt{\frac{1}{M_{\mathrm{eff}}}},
$$

where $v_{0}$ is the most likely velocity at $293.6 \mathrm{~K}$ and $2200 \mathrm{~m} / \mathrm{s}$.

The absorption interaction frequency $I_{a}$ is obtained with

$$
I_{a}=v \Sigma_{a} .
$$

The program then computes a random time-to-interaction $t$ from a uniformly drawn value $r$ between 0 and 1 using

$$
t=\frac{-\ln r}{I_{a}+I_{s}}
$$

and from this time $t$ and the velocity of the neutron the point of interaction in space follows. If this point lies within the object, a scattering or absorption event has occurred, and a second random number is drawn to decide which. If the point 


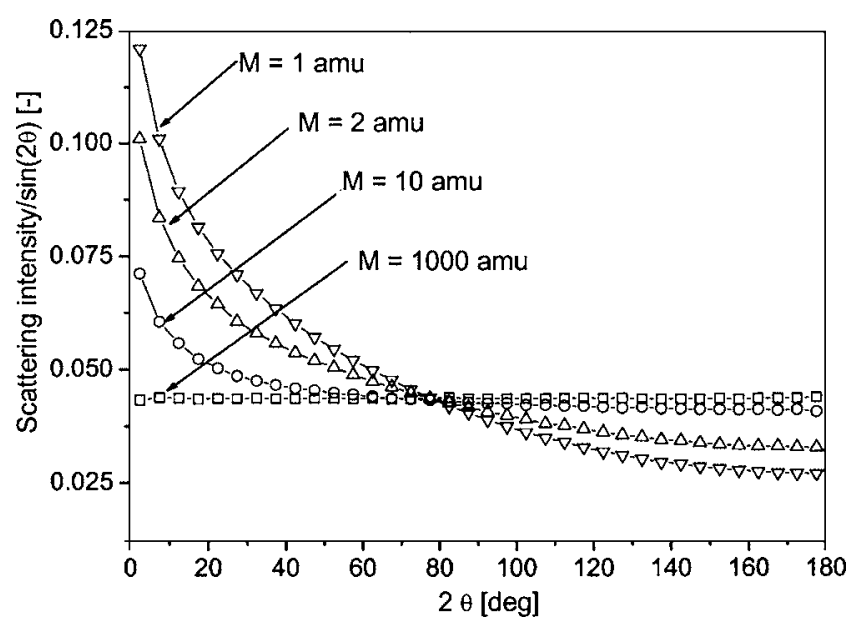

FIG. 2. Simulated scattering intensity as a function of scattering angle for various masses for a "free-gas" scatterer at room temperature for a neutron spectrum as employed at the NIST NG0 facility. The scattering yield is normalized to the total number of neutrons employed in the simulation. The figure shows that the scattering is isotropic at infinite mass $(M=1000)$ and becomes anisotropic as the mass decreases.

lies beyond the object, the process is repeated with unchanged velocity and the exit point as the new starting point.

To calculate the neutron velocity and direction in the case of a scattering event, the velocity of the scattering atom is drawn from the Maxwell-Boltzmann distribution mentioned in Eqs. (8) and (9), with a random direction, using the methods described in the MCNP documentation. In the centerof-mass frame, the scattering is considered isotropic so the new direction of the neutron is drawn at random in that frame. Employing the laws of conservation of momentum and energy the neutron velocity is obtained. Finally, the velocity is translated back to the laboratory frame.

\section{E. Example simulations}

The BUDA program was employed to simulate the interaction between a neutron beam as present at the NG0 facility at NIST, and a scatterer described by the free-gas model. The scatterer temperature and mass were varied and the effect on the isotropy was observed. The output was divided in bunches of $2.5^{\circ}$ from $0^{\circ}$ to $180^{\circ}$ and consisted of the number of neutrons scattered within each particular angle interval.

Figure 2 presents the scattering intensity as a function of scattering angle for a free-gas scatterer for various masses at a constant temperature of $298 \mathrm{~K}$. The yield is normalized to the total number of neutrons employed in the simulation. For clarity of representation, the scattering intensity is reported divided by the sine of the corresponding angle. As expected, for an infinite mass $(M=1000)$, the scattering is isotropic; for decreasing mass, the signal gets increasingly anisotropic; in particular, the forward signal increases at the expense of the backward signal.

In Fig. 3 the simulated scattering intensity as a function of angle for various scatterer temperatures is shown. Also in this figure the yield is normalized to the total number of neutrons and divided by the sine of the scattering angle. The scatterer employed has $M=1$. It can be observed that the

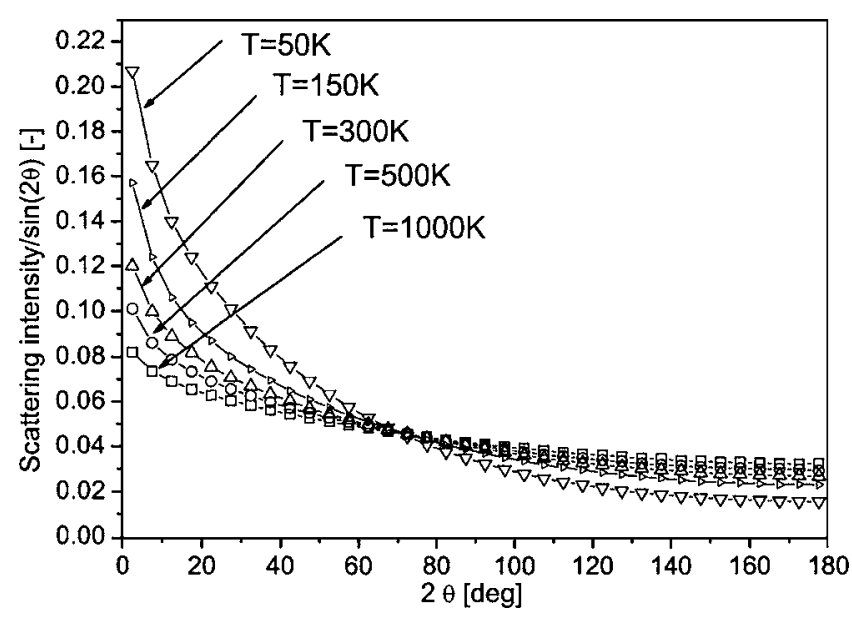

FIG. 3. Simulated scattering intensity as a function of scattering angle for various scatterer temperatures for a "free-gas" scatterer with $M=1$ and a neutron spectrum as employed at the NIST NG0 facility. The scattering yield is normalized to the total number of neutrons employed in the simulation. The figure shows that the scattering anisotropy increases with decreasing temperature.

isotropy increases with increasing temperature. Total isotropy is not obtained in this simulation, since the maximum temperature value is limited.

The scattering of a thin hydrogen slab as employed in the NIS experiments as a function of hydrogen effective mass at angles of $45^{\circ}$ and $135^{\circ}$ was simulated as well. The macroscopic scattering cross section for each hydrogen effective mass was calculated with Eq. (5). The value used for $\sigma_{\text {free }}$, the total scattering cross section of the free hydrogen atom was $20.5 \mathrm{~b}$. This value was adjusted for each effective mass using Eq. (3). The value for the absorption cross section was $0.3326 \mathrm{~b}$. ${ }^{4}$ The pure hydrogen slab model was considered to be applicable because a titanium blank was available, so that net hydrogen scattering intensities could be obtained, and it was considered to be thin because the hydrogen concentrations in the sample were known to be low. The scattering intensity relative to "rigidly bound" for the two angles of $45^{\circ}$ and $135^{\circ}$ (left $y$ axis) and the ratio of the two intensities (right $y$ axis), as a function of hydrogen effective mass, are presented in Fig. 4. The scattering intensities are normalized to the scattering intensity for bound hydrogen, i.e., modeled with effective mass of 1000. The graph shows a complex relationship between the scattering at the two angles and the hydrogen effective mass; the forward/ backward ratio, however, is a monotonically decreasing function of the hydrogen effective mass.

\section{F. The free-gas NIS experiment}

As opposed to isotropic NIS, a fundamental part of the free-gas NIS experiments consists of data analysis based on Monte Carlo simulations with the free-gas model employing the BUDA program described above.

A free-gas NIS experiment thus consists of the following steps:

(1) NIS experiment with neutron detection at two scattering angles.

(2) Monte Carlo simulations with the BUDA program em- 


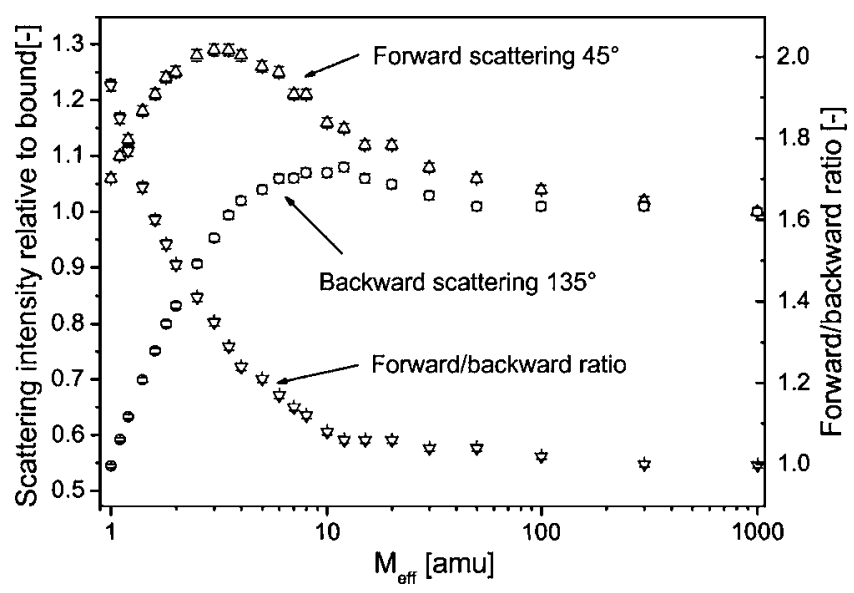

FIG. 4. Simulated scattering intensities as a function of hydrogen effective mass for the angles $45^{\circ}$ and $135^{\circ}$ employed in the free-gas NIS experiments. The left $y$ axis refers to the scattering intensity, whereas the right $y$ axis refers to the forward/backward ratio. The forward and backward scattering reveal a complex dependence on the hydrogen effective mass, the forward to backward ratio, however, is a monotonically decreasing function of the effective mass.

ploying the free-gas model, providing the calibration which allows for the determination of the hydrogen effective mass in the sample analyzed. This simulation is specific for the sample matrix composition as well as for its dimensions.

(3) Determination of the hydrogen effective mass from the ratio of the two observed scattering intensities.

(4) The observed effective mass translates to the scattering cross sections in the two directions, so that the scattering intensities can be quantitatively interpreted in terms of hydrogen content.

This process may have to be iterated especially at high hydrogen concentrations, since the scattering intensity ratio is also affected by multiple scattering in the sample.

\section{RESULTS}

\section{A. CNPGAA}

Table I represents the results of CNPGAA on all samples analyzed. For comparison, the measurements performed in 1998 shortly after preparation ${ }^{6}$ are listed as well. In the fol-

TABLE I. Nominal and measured (1998 and 2002) hydrogen concentration in the titanium alloy samples.

\begin{tabular}{ccc}
\hline \hline & $\begin{array}{c}\text { Concentration CNPGAA } \\
{[\mathrm{mg} / \mathrm{kg}]}\end{array}$ & $\begin{array}{c}\text { Concentration CNPGAA } \\
{[\mathrm{mg} / \mathrm{kg}]}\end{array}$ \\
{$\left[\begin{array}{c}\text { Nominal concentration } \\
{[\mathrm{mg} / \mathrm{kg}]}\end{array}\right.$} & {$[1988]$} & $0 \pm 5]$ \\
\hline 0 & $0 \pm 8$ & $87 \pm 7$ \\
92.3 & $86 \pm 3$ & $171 \pm 11$ \\
208 & $173 \pm 6$ & $246 \pm 12$ \\
238 & $239 \pm 6$ & $257 \pm 11$ \\
275 & - & $273 \pm 12$ \\
306 & $253 \pm 7$ & $375 \pm 16$ \\
375 & $382 \pm 8$ & \\
\hline \hline
\end{tabular}

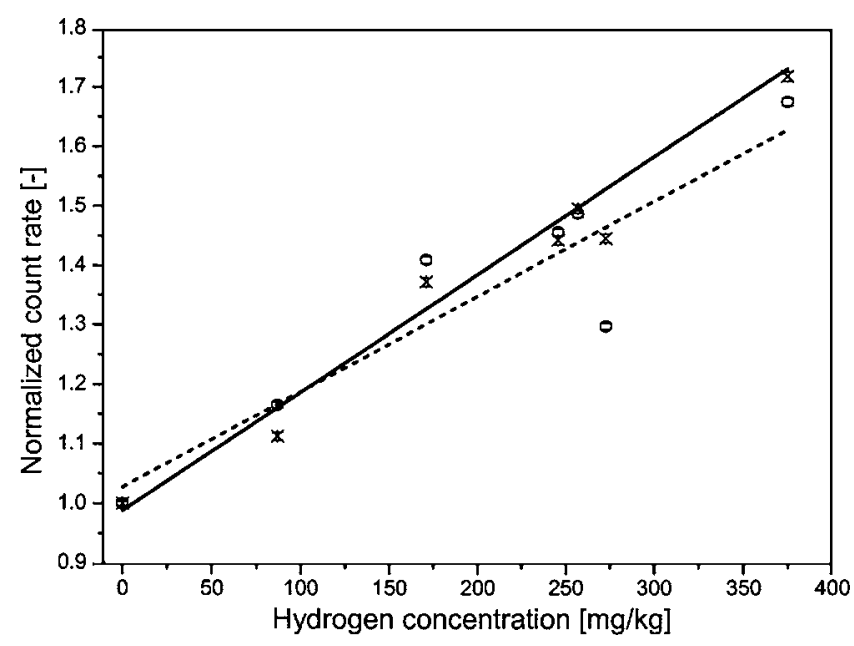

FIG. 5. Forward $\left(45^{\circ}, X\right)$ and backward $\left(135^{\circ}, O\right)$ scattering count rate for experiment 4 (see Table II). Linear curves were drawn to guide the eye: a solid line for forward scattering and dotted line for backward scattering. The $273-\mathrm{mg} / \mathrm{kg}$ sample shows lower backward scattering, resulting in high anisotropy.

lowing, the hydrogen-in-titanium samples are referred to by employing the concentrations as measured by CNPGAA in 2002, i.e., the third column in Table I.

\section{B. Free-gas NIS \\ 1. NIS experiments}

A typical result of the NIS measurements on the titanium samples is shown in Fig. 5. The NIS-normalized count rates for forward $\left(45^{\circ}\right)$ and backward $\left(135^{\circ}\right)$ scattering are plotted versus the hydrogen concentration obtained by CNPGAA. The results agree with the measurements performed earlier by Blaauw, ${ }^{6}$ with the $273-\mathrm{mg} / \mathrm{kg}$ sample yielding an anomalous result again. This sample was therefore treated separately during data analysis and in the following discussion.

The experimental values were fitted with a linear equation of the form

$$
y=(1+a x) b,
$$

where $a\left([\mathrm{mg} / \mathrm{kg}]^{-1}\right)$, i.e., the slope-over-intercept ratio of the straight lines in Fig. 5, represents the increase in hydrogen scattering relative to the titanium scattering and $b$, i.e., the intercept, represents the titanium scattering. With the parameter $a$, the apparent hydrogen scattering cross section in the direction of interest can be calculated with the following equation:

$$
\sigma_{\mathrm{H}}=a \times 1 \times 10^{6} \times \frac{M_{\mathrm{H}}}{M_{\mathrm{Ti}}} \times \sigma_{\mathrm{Ti}}[\mathrm{b}] .
$$

The factor $1 \times 10^{6}$ accounts for the hydrogen concentration being expressed in $\mathrm{mg} / \mathrm{kg}$. $\sigma_{\mathrm{Ti}}=2.79 \mathrm{~b}$, the scattering cross section per atom in the titanium alloy, has been calculated employing the incoherent scattering cross sections for the elements $\mathrm{Ti}, \mathrm{Al}$, and $\mathrm{V}$ reported in Ref. 4.

Data obtained in four experiments for all samples except the $273 \mathrm{mg} / \mathrm{kg}$ sample were fitted simultaneously. The data for the $273-\mathrm{mg} / \mathrm{kg}$ sample were treated separately. Both results are presented in Table II. The table includes the slope, 
TABLE II. Values for the $a$ parameter, i.e., the slope over intercept ratio, and for $\sigma_{\mathrm{H}}$ for four experiments at the two scattering angles.

\begin{tabular}{|c|c|c|c|c|c|c|c|c|}
\hline \multicolumn{5}{|c|}{ Forward scattering $\left(45^{\circ}\right)$} & \multicolumn{4}{|c|}{ Backward scattering $\left(135^{\circ}\right)$} \\
\hline Expt. & a & Error & $\left(\sigma_{\mathrm{H}}\right)_{45^{\circ}}$ & Error & a & Error & $\left(\sigma_{\mathrm{H}}\right)_{135^{\circ}}$ & Error \\
\hline \multicolumn{9}{|c|}{ All samples except $273 \mathrm{mg} / \mathrm{kg}$} \\
\hline 1 & $1.67 \times 10^{-3}$ & $6.91 \times 10^{-3}$ & 97 & 4 & $1.52 \times 10^{-3}$ & $1.22 \times 10^{-4}$ & 88 & 7 \\
\hline 2 & $1.31 \times 10^{-3}$ & $1.09 \times 10^{-4}$ & 76 & 6 & $1.27 \times 10^{-3}$ & $1.70 \times 10^{-4}$ & 74 & 10 \\
\hline 3 & $1.45 \times 10^{-3}$ & $2.56 \times 10^{-4}$ & 84 & 15 & $1.42 \times 10^{-3}$ & $2.50 \times 10^{-4}$ & 82 & 15 \\
\hline 4 & $1.98 \times 10^{-3}$ & $1.70 \times 10^{-4}$ & 115 & 10 & $1.83 \times 10^{-3}$ & $1.71 \times 10^{-4}$ & 106 & 10 \\
\hline Mean & $1.62 \times 10^{-3}$ & $5.39 \times 10^{-5}$ & 94 & 3 & $1.53 \times 10^{-3}$ & $8.10 \times 10^{-5}$ & 89 & 5 \\
\hline \multicolumn{9}{|c|}{ Sample $273 \mathrm{mg} / \mathrm{kg}$} \\
\hline 1 & $1.54 \times 10^{-3}$ & $1.68 \times 10^{-5}$ & 90 & 1 & $8.52 \times 10^{-4}$ & $1.91 \times 5^{-}$ & 50 & 1 \\
\hline 2 & $1.23 \times 10^{-3}$ & $2.80 \times 10^{-5}$ & 71 & 2 & $9.05 \times 10^{-4}$ & $3.82 \times 10^{-5}$ & 53 & 2 \\
\hline 3 & $1.46 \times 10^{-3}$ & $1.23 \times 10^{-5}$ & 85 & 1 & $1.08 \times 10^{-3}$ & $1.05 \times 10^{-5}$ & 63 & 1 \\
\hline 4 & $1.63 \times 10^{-3}$ & $2.40 \times 10^{-5}$ & 95 & 1 & $1.09 \times 10^{-3}$ & $1.92 \times 10^{-5}$ & 63 & 1 \\
\hline Mean & $1.49 \times 10^{-3}$ & $8.71 \times 10^{-5}$ & 86 & 1 & $1.04 \times 10^{-3}$ & $8.09 \times 10^{-6}$ & 60 & 0.5 \\
\hline
\end{tabular}

$a$, and the calculated hydrogen scattering cross section, $\sigma_{s}$, both for the forward and the backward scattering. The last row represents the weighted mean of the four experiments.

\section{Free-gas Monte Carlo simulation and data analysis}

The values obtained from the Monte Carlo simulation in Fig. 4 were employed to interpret the experimental results presented in Table II in terms of hydrogen effective mass. The outcome is shown in Table III. The columns $\left(\sigma_{\mathrm{H}}\right)_{45^{\circ}} / \sigma_{\text {bound }}$ and $(\sigma \mathrm{H})_{135^{\circ}} / \sigma_{\text {bound }}$ represent the appropriate mean values in Table II divided by the scattering cross section of the bound hydrogen atom (80.3 b). The next column represents their ratio. In the following three columns, the same values for the Monte Carlo simulations, as obtained in Fig. 4, are shown. The free-gas analysis performed by deriving an effective mass of 12 from the forward/backward ratio and then using the corresponding theoretical cross sections in either direction would have yielded the correct hydrogen concentrations for all samples, without the use of standards, except for the $273-\mathrm{mg} / \mathrm{kg}$ sample.

For the $273-\mathrm{mg} / \mathrm{kg}$ sample no satisfactory match could be found between the experiment and simulation. It was then hypothesized that two states of hydrogen might be present simultaneously. A fraction $f$ and two effective-mass values were found such that the following equation was satisfied in both scattering directions:

$$
\begin{aligned}
\left(\sigma_{\mathrm{H}} / \sigma_{\text {bound }}\right)_{\text {measured }}= & f\left[\sigma_{\mathrm{H}}\left(\theta, M_{1}\right) / \sigma_{\text {bound }}\right]+(1-f) \\
& \times\left[\sigma_{\mathrm{H}}\left(\theta, M_{2}\right) / \sigma_{\text {bound }}\right] .
\end{aligned}
$$

The solution that satisfied this condition was $f=0.625, M_{1}$ $=1.2$, and $M_{2}=\infty$.

\section{DISCUSSION}

The NIS and CNPGAA measurements performed in 1998 (Ref. 6) were repeated in 2002 in order to verify if the hydrogen concentration and the NIS response remained constant. In particular, in 1998 a different scattering behavior was observed in the $273-\mathrm{mg} / \mathrm{kg}$ sample and it was thought that this might be due to higher hydrogen mobility, perhaps leading to hydrogen loss over time.

The experiments performed in 2002 (Fig. 5) exhibit the same trend as those performed previously, the PGNAA results being statistically identical, meaning that the hypothesis of hydrogen loss over time in Ref. 6 was falsified. The final results in terms of observed effective masses and scattering cross sections agree with the known sample compositions for the "normal" samples, i.e., the $273-\mathrm{mg} / \mathrm{kg}$ sample excluded.

TABLE III. Comparison of experimental results with Monte Carlo simulation. The table shows the scattering cross section normalized to bound scattering

\begin{tabular}{|c|c|c|c|c|c|c|c|}
\hline & $\begin{array}{c}\left(\sigma_{\mathrm{H}}\right)_{45^{\circ}} / \\
\sigma_{\text {bound }}\end{array}$ & $\begin{array}{c}\left(\sigma_{\mathrm{H}}\right)_{135^{\circ}} / \\
\sigma_{\text {bound }}\end{array}$ & $\begin{array}{l}\left(\sigma_{\mathrm{H}}\right)_{45^{\circ}} / \\
\left(\sigma_{\mathrm{H}}\right)_{135^{\circ}}\end{array}$ & $\begin{array}{c}\left(\sigma_{\mathrm{H}}\right)_{45^{\circ}} / \\
\sigma_{\text {bound }} \\
\text { Monte Carlo }\end{array}$ & $\begin{array}{c}\left(\sigma_{\mathrm{H}}\right)_{135^{\circ}} / \\
\sigma_{\text {bound }} \\
\text { Monte Carlo }\end{array}$ & $\begin{array}{c}\left(\sigma_{\mathrm{H}}\right)_{45^{\circ}} / \\
\left(\sigma_{\mathrm{H}}\right)_{135^{\circ}} / \\
\text { Monte Carlo }\end{array}$ & $\begin{array}{c}\mathrm{M}_{\mathrm{eff}} \\
{[\mathrm{amu}]}\end{array}$ \\
\hline $\begin{array}{l}\text { Mean all excl. } \\
273 \mathrm{mg} / \mathrm{kg}\end{array}$ & $1.17 \pm 0.04$ & $1.11 \pm 0.06$ & $1.06 \pm 0.07$ & $1.15 \pm 0.008$ & $1.08 \pm 0.008$ & $1.06 \pm 0.008$ & 12 \\
\hline $\begin{array}{c}\text { Mean } \\
273 \mathrm{mg} / \mathrm{kg}\end{array}$ & $1.08 \pm 0.01$ & $0.75 \pm 0.01$ & $1.43 \pm 0.01$ & $1.08 \pm 0.01$ & $0.77 \pm 0.008$ & $1.40 \pm 0.02$ & $\begin{array}{c}1.2(62.5 \%) \\
\infty(37.5 \%)\end{array}$ \\
\hline
\end{tabular}
cross section for the experiments performed on the titanium samples (Table II) and for the Monte Carlo simulations (Fig. 4). 
That is, the free-gas NIS approach yields the correct hydrogen concentrations in these samples, and that without the use of a calibration sample.

The $273-\mathrm{mg} / \mathrm{kg}$ specimen clearly shows a different behavior, suggesting that it differs from the others not only in hydrogen concentration but also in hydrogen state or structure. The anomalous sample showed lower backward scattering and a clearly anisotropic response. An influence on the measurement of oscillations in the ambient temperature was excluded, since the NIS results were completely reproducible at various times of the day. Also, as already mentioned, the outlier behavior was observed in the earlier experiments as well. ${ }^{6}$

As discussed in the theoretical description of free-gas NIS, anisotropy may be correlated with hydrogen effective mass and thus hydrogen binding in the sample. Analysis of the NIS data with the free-gas model showed that the experimental data resulted in a hydrogen effective mass of $12 \mathrm{amu}$ for the normal titanium chips, whereas for the $273 \mathrm{mg} / \mathrm{kg}$, the free-gas model seemed to indicate that this sample had a somewhat more complex composition consisting of two groups of hydrogen, one with effective mass of $1.2 \mathrm{amu}$ and the second one with infinite effective mass, i.e., tightly bound (Table III). The physical interpretation of these findings is not obvious; the lower effective mass of a fraction of the hydrogen in the $273-\mathrm{mg} / \mathrm{kg}$ sample might represent higher hydrogen mobility, meaning that at a given point in time, some hydrogen atoms are freely moving through the crystal lattice, while others are tightly bound and cannot exchange kinetic energy with the slow neutrons.

It was discovered that, in order for isotropic NIS to yield correct results, the standard should not only consist of the same material as the unknown sample, as stated in Ref. 5, but also have comparable hydrogen effective mass. In the case of polypropylene, the hydrogen effective mass may be approximated by the one of polyethylene, which has been determined experimentally by measuring the average cosine of the scattering angle. This was found to be 0.1, which corresponds to a hydrogen effective mass of 7 amu [see Eq. (4)]. ${ }^{14}$ This indicates that the hydrogen is moderately tightly bound to the matrix and use of polypropylene as a standard for materials in which (some of) the hydrogen might not be as tightly bound yields erroneous results depending on the observation angle. Detection of neutrons scattered under two different angles is thus necessary as a safeguard against unexpected variations in anisotropy.

\section{CONCLUSIONS}

A first attempt has been made to get a more thorough understanding of the NIS measurements.

It was demonstrated that, in order to apply NIS for the determination of hydrogen concentration in metal alloys, a more complex model than the isotropic one needs to be employed that takes into account the influence of chemical binding and sample temperature on the apparent scattering cross section of hydrogen. In particular, it was shown that determining the hydrogen concentration in a sample with unknown effective hydrogen mass by the free-gas NIS procedure yields the correct result in most cases without requiring the use of standards; however, there could be cases, such as the $273-\mathrm{mg} / \mathrm{kg}$ sample, where the procedure would yield erroneous results.

Further research is required to establish a link between hydrogen effective mass and hydrogen chemical state in the matrix. It is suggested that this could be useful for applications in material science.

\section{ACKNOWLEDGMENTS}

The Netherlands Organization of Scientific Research (NWO) is acknowledged for funding the stay of one of the authors (R.C.P.) at NIST. H. H. Chen-Mayer, G. Lamaze, D. Mildner, and R. M. Lindstrom are acknowledged for the experimental help and for the useful discussions.

${ }^{1}$ N. E. Paton and J. C. Williams, in Titanium and Titanium Alloys: Source Book, edited by M. J. Donachie, Jr., (American Society for Metals, Metals Park, OH, 1982).

${ }^{2}$ H. H. Chen-Mayer, D. F. R. Mildner, G. P. Lamaze, R. M. Lindstrom, R. L. Paul, V. V. Kvardakov, and W. J. Richards, Mater. Res. Soc. Symp. Proc. 513, 191 (1998).

${ }^{3}$ V. V. Kvardakov, H. H. Chen-Mayer, D. F. R. Mildner, and V. A. Somenkov, J. Appl. Phys. 83, 3876 (1998).

${ }^{4}$ V. F. Sears, Neutron News 3, 29 (1992).

${ }^{5}$ Y. N. Choi, H. S. Oh, V. T. Em, V. A. Somenkov, C.-H. Lee, and S. D. Park, Appl. Phys. A 74, S1710 (2002).

${ }^{6}$ M. Blaauw, Nucl. Instrum. Methods Phys. Res. A 505, 20 (2003).

${ }^{7}$ R. M. Lindstrom and R. L. Paul, Mater. Res. Soc. Symp. Proc. 513, 283 (1998).

${ }^{8}$ E. Fermi, Nuclear Physics (University of Chicago Press, Chicago, 1950).

${ }^{9}$ K. H. Beckurts and K. Wirtz, Neutron Physics (Springer, Berlin, 1964).

${ }^{10}$ E. Fermi, Ric. Sci. 7, 13 (1936).

${ }^{11}$ J. F. Briesmeister, Report No. LA-12625 (Los Alamos, March, 1997).

${ }^{12} \mathrm{M}$. Blaauw, BUDA.

${ }^{13}$ D. F. R. Mildner, H. H. Chen-Mayer, G. P. Lamaze, and V. A. Sharov, Nucl. Instrum. Methods Phys. Res. A 413, 341 (1998).

${ }^{14}$ J. R. Granada, J. Dawidowski, R. E. Mayer, and V. H. Gilette, Nucl. Instrum. Methods Phys. Res. A 261, 573 (1987). 